\title{
El capital cultural del profesorado en los procesos de inclusión educativa
}

\author{
The teaching cultural capital in the educational inclusion processes
}

\author{
Manuel Gregorio Ortiz Huerta \\ Mauricio Zacarías Gutiérrez
}

\begin{abstract}
RESUMEN
El presente ensayo plantea el capital cultural que tienen los profesores como detrimento de prácticas inclusivas en el espacio escolar cuando componentes pedagógicos como pedagogía, escuela y escolaridad no consideran la diversidad del estudiante. Las prácticas culturales, el sentido y significado de la escuela y la obligatoriedad de la educación en el sistema neoliberal resultan mecanismos que son incompatibles a los procesos de inclusión del estudiante en la escuela; el capital cultural del profesor instituye una pedagogía conservadora que es lineal al currículo y al sistema político-pedagógico neoliberal pues resulta eficaz a los intereses de dicho sistema, el capital cultural del profesorado instituido en prácticas culturales conservadoras forma un habitus en el estudiante que inhibe su presencia, participación y pertenencia; por ello, se requiere la reflexión de la práctica educativa desde una pedagogía de las diferencias que otorgue sentido y significado a las experiencias del otro y se construya socialmente la alteridad.
\end{abstract}

Palabras clave: capital cultural, escuela, escolaridad obligatoria.

\section{Abstract}

This essay raises the cultural capital issue that teachers face as a detriment of inclusive practices in school when pedagogical elements such as pedagogy, school and schooling do not consider student diversity. Cultural practices, the meaning and significance of school and the mandatory of education in the neoliberal system are mechanisms that are incompatible with the processes of inclusion of the student in school; the cultural capital of the teacher founds a conservative pedagogy that is linear to the curriculum and the neoliberal political-pedagogical system because it is effective for the interests of said system. The cultural capital of teachers created by conservative cultural practices forms a babitus in the student that inhibits their presence, participation and belonging; therefore, reflection on educational practice is required from a pedagogy of differences that gives meaning and significance to the experiences of the other and socially constructs otherness.

Keywords: cultural capital, school, mandatory schooling. 


\section{INTRODUCCIÓN}

El presente ensayo surge a partir de la reflexión sobre el capital cultural de profesores y la dificultad para implementar una educación inclusiva dada la formación académica de prácticas mecanizadas en la trasmisión del conocimiento, en actividades pedagógicas rutinarias y la inconsciencia del estudiante en cuanto a un sujeto de inclusión. La práctica educativa del profesor que asume la inclusión en la escuela será referente de prácticas sociales democráticas, así como de orientaciones ideológicas sobre la diversidad del estudiante y la construcción social de la alteridad. Por tanto, se reflexiona si las prácticas educativas vividas en el aula son actividades democráticas; de igual manera, hasta dónde se considera la diversidad del estudiante y cómo pueden evitarse acciones pedagógicas de exclusión y segregación, esto desde la comprensión de los procesos de inclusión en el sistema neoliberal actual que permean las políticas educativas.

Díaz (2002) enuncia que la práctica educativa basada en la didáctica tradicional enfatiza la actividad de enseñanza sobre la actividad de aprendizaje del estudiante, es un proceso lineal, por ello, no hay atención sobre el estudiante ni por sus intereses, curiosidades o problemas, se trabaja la memoria verbal reproductiva, por ende hay una exclusión de la palabra, así como un intelectualismo exagerado; en este sentido, el trabajo académico está en correspondencia a la lógica del sistema imperante que evidencia una clara pedagogía conservadora y simplicidad del acto pedagógico, en la cimentación de una educación bancaria (Freire, 2017). Giroux (2006) identifica la pedagogía conservadora como macro-categoría de la pedagogía que da cuenta de un proceso educativo reduccionista al imperar el conocimiento universal, en la cual lo educativo se estructura en la hegemonía de la ideología dominante. Las prácticas educativas de una pedagogía conservadora aparecen en la interrelación entre profesores y estudiantes en el aula y tienden a ser un reflejo del sistema socioeconómico de sociedades neoliberales; las prácticas educativas sustentadas en una racionalidad técnica

Manuel Gregorio Ortiz Huerta. Profesor-investigador de la Escuela Normal Fray Matías de Córdova, Chiapas, México. Cuenta con estudios de licenciatura en Pedagogía, maestría en Docencia y doctorando en Educación. Es docente de posgrado en instituciones públicas y privadas. Entre sus publicaciones recientes se encuentra "Hacia una fenomenología del alumno en la escuela y vida escolar" (2021). Pertenece a la Red Durango de Investigadores Educativos. Actualmente se encuentra en el desarrollo de investigación educativa sobre el discurso de la identidad profesional docente en estudiantes normalistas de la licenciatura en Inclusión Educativa. Correo electrónico: manuelhuerta31101982@gmail.com. ID: https://orcid.org/0000-0003- 3977-0953.

Mauricio Zacarías Gutiérrez. Profesor-investigador del Centro Regional de Formación Docente e Investigación Educativa, México. Cuenta con estudios de licenciatura en Educación Primaria, maestría en Educación Superior y doctorado en Estudios Regionales. Pertenece a la Red Durango de Investigadores Educativos y a la Red Mexicana de Investigadores de la Investigación Educativa. Entre las investigaciones realizadas ha cultivado la formación docente, tanto inicial como continua, así mismo el saber docente de profesores que laboran en la educación especial. Actualmente se encuentra desarrollando investigación educativa relacionada al afecto docente en la educación primaria. Correo electrónico: mazag@hotmail.com. ID: https://orcid.org/00000003-4564-5673. 
(modelo educativo neoliberal) suelen desarrollar actividades pedagógicas rutinarias en el aula que adoctrinan al estudiante en competencias básicas para dicho sistema; el aula es el lugar en donde se concreta el currículo, se construyen identidades y se manifiesta la cultura escolar. Desde un sentido de la teoría crítica en la educación, la escuela y el currículo resultan un dispositivo sociocultural de exclusión al seleccionar y clasificar al estudiante porque reducen su formación a un sentido lineal; en este sentido, la linealidad tergiversa la diversidad a procesos académicos homogéneos.

Por otro lado, la escolarización se instituye en la sociedad como una política social en una educación obligatoria; la escolarización del sistema educativo tensa lo educativo e institucional porque la naturaleza educativa corresponde a una dimensión política que el profesor considera en su pedagogía (práctica educativa) en la habitualidad del desarrollo del currículo. La tensión entre lo educativo e institucional ocurre en los procesos de calidad educativa (eficiencia y eficacia) porque para lograr los fines educativos se precisa de tecnificar el proceso académico y didáctico, lo que remite la selectividad de competencias, la clasificación del conocimiento y la evaluación de los procesos de aprendizaje, ello trae como consecuencia la exclusión y segregación del estudiante cuando este no alcanza los estándares educativos, se aparta de quienes sí cumplen (etiqueta de insuficiencia), dicha situación conlleva a normalizar la selección y clasificación de competencias. Las sociedades escolarizadas se caracterizan en la educación por la capacitación, formación y selección de capital humano (instrumentación de la educación), por tanto, es necesario repensar si la práctica educativa reproduce las políticas neoliberales de escolarización cuando se busca implementar prácticas inclusivas.

Se analiza la correlación transversal de la acción pedagógica del profesorado que tiene en la escuela y que desarrolla en el marco de la educación obligatoria, porque socialmente se induce la educabilidad de los sujetos a un estatus moral, a la distinción de ser escolarizado; la escuela es un espacio social en el que se forma el capital cultural del profesorado, a su vez, el capital cultural remite una formación académica en los estudiantes; así pues, el capital cultural es una condición del habitus del estudiante que precisa de condiciones sociales y culturales como lo es la educación obligatoria.

\section{INCLUSIÓN EDUCATIVA Y CAPITAL CULTURAL}

Actualmente, los sistemas educativos bajo el imperativo filosófico y económico del neoliberalismo plantean la inclusión en las instituciones educativas como un modelo alternativo a la desigualdad y exclusión social. La inclusión como fenómeno social forma parte del discurso de la política global de la Organización de las Naciones Unidas; la inclusión educativa es un proceso de desarrollo integral en el que participan agentes de la comunidad educativa (Sánchez, 2018). La inclusión tiene como propuesta concebir al estudiante como sujeto de derecho, su permanencia en la escuela y el 
derecho a una educación de calidad, además se busca contrarrestar una educación fincada de individualidad, fricciones, rivalidades, esfuerzo personal sobre el trabajo colectivo y bien común (Martínez, 2014), es decir, que se modifique la mecanización de la enseñanza (trasmisión del conocimiento), que se sustenten las actividades pedagógicas en y desde la otredad y alteridad, fomentar la enseñanza de valores para la convivencia democrática y eliminar la desigualdad, exclusión y segregación.

La inclusión en la educación requiere de una escuela para la convivencia democrática, esto es, que la escuela esté en una línea de responsabilidad ciudadana, para ello se precisa de apertura interna y búsqueda de colaboración y colegialidad profesional, afuera la escuela tendrá una visión comunitaria (Fernández, 2002, citado en García, 2006). La inclusión en lo educativo alude a prácticas sociales democráticas en las cuales la participación social de profesores construye el sentido de justicia, de igual forma se busca tener experiencias colectivas para mediar conflictos que puedan suscitarse en las aulas, tales como violencia, discriminación, racismo, desigualdad, exclusión, entre otros.

La inclusión en los procesos educativos está sustentada teóricamente en una pedagogía que considera la formación integral del sujeto en su alteridad, es decir, pensar la unidad en la diversidad (Freire, 2017). Bartolomé y Cabrera (2007) refieren una pedagogía de las acciones formativas en la que se oriente la enseñanza hacia los procesos de calidad del aprendizaje de estudiantes en una educación "independiente del origen racial, procedencia cultural, género o preferencia sexual, clase social, etc." (p. 60). La inclusión educativa toma en cuenta las características individuales del estudiante, como su personalidad y cultura; aborda las diferencias en el aula y los estilos de aprendizaje. Farrel (2001, citado en Dueñas, 2010) señala que la inclusión educativa se relaciona con la aceptación de todos en cuanto miembros de pleno derecho del grupo y se valora la contribución que tienen, destacando el derecho de participación de la persona en sociedad.

El objetivo de la inclusión educativa es transformar los procesos formativos para superar la desigualdad, que es eje central de la educación inclusiva (Sánchez, 2018); la inclusión social es una alternativa a situaciones de exclusión y segregación del sujeto en sociedades neoliberales, emerge como disyunción a la imposición ideológica de la educación centrada en la tecnificación educativa y la racionalidad instrumental. La educación inclusiva conforma un criterio político-pedagógico de educación para todos con miras al desarrollo de una cultura de tolerancia a lo diferente, al resguardo de los derechos humanos y a la igualdad de oportunidades (Montanchez, 2015). Por otro lado, la educación en tiempos neoliberales está fuertemente compenetrada, influida por factores económicos que mercantilizan el sistema educativo, privatizan la educación, reducen la educación a un componente de consumo; esto ha provocado una exclusión del sujeto en sociedades mercantiles o industrializadas al no contar con las competencias requeridas para dicho tipo de educación (Torres, 2007). 
El discurso educativo en el neoliberalismo (que se sacralizó por los capitalistas) indujo un enfoque mercantilista en los procesos educativos en el que se priorizó la formación de capital humano sobre el desarrollo humano, ello suscitó en la educación el cambio de paradigma del humanismo hacia la competitividad, a su vez, se implantó la ideología de la sociedad competitiva (De la Torre, 2004). En eco de lo anterior, existe la discusión sobre la implementación de la teoría curricular que reivindique el humanismo en la educación de sociedades neoliberales porque los modelos curriculares conciben la educación como instrumento de desarrollo económico, con ello la instauración de proyectos curriculares; además se toma en cuenta el papel y organización de las instituciones educativas plasmadas en la racionalidad instrumental (Martínez, 1998).

En las sociedades industrializadas, la educación constituye un indicador de progreso social y económico, la educación de las masas soporta una amalgama de teorías educativas que abordan la relación entre escolarización, currículo y desarrollo social o progreso de la sociedad (Kemmis, 2008). En el sentido político, la derecha o partidos conservadores etiológicamente refieren que los aspectos sociales y educativos son el mal de las sociedades, es decir, que los males sociales requieren de corregirse en el fortalecimiento ante las debilidades de los individuos (Beyer y Liston, 2001).

La relación entre escuela, currículo y sociedad es determinada por las políticas sociales conservadoras y de derecha ideológica en que se implementan proyectos educativos como la escolarización de grupos vulnerables para la solución de los problemas sociales; en este orden se propusieron conocimientos comunes tanto para lo social como lo textual, así como un currículo multicultural que destacara las diferencias en vez de las similitudes (Beyer y Liston, 2001), sin embargo, el planteamiento del currículo multicultural conllevó al debate multicultural: por un lado, pluralistas indicaron una propuesta de cohesión, mientras que los particularistas refirieron un futuro dividido y antagónico (Ravitch, 1990, citado en Beyer y Liston, 2001).

Se piensa entonces en que sociedades escolarizadas disminuirán los problemas sociales y que los modelos multiculturales compaginados con filosofías económicas como el liberalismo clásico desarrollarán la economía en las sociedades, esto a partir de estimar "dimensiones filosóficas, sociales, religiosas, morales, políticas y económicas, que constituyen una visión del mundo de importantes repercusiones para la vida cotidiana" (Beyer y Liston, 2001, p. 65), no obstante, los procesos educativos contemporáneos requieren de un discurso concreto, práctico, y no un agotamiento de la práctica en cuanto a la extensión del conocimiento y su complejidad teórica (Freire, 2019).

La educación en los modelos neoliberales destaca la inversión del capital humano y su relación al desarrollo económico, en la que la educación es tratada como mercancía (Villalobos y Pedroza, 2009). A partir del análisis de la teoría del capital humano y su obstinada idea de desarrollo económico, los modelos pedagógicos como la inclusión 
educativa han pugnado por un enfoque humanista dada la homogeneidad de procesos formativos académicos, el establecimiento de conocimiento comunes y educar bajo pretensiones desarrollistas globales; en cierta forma, estos procesos reducen la educación a un sistema mecanicista de trasmitir habilidades y competencias básicas que adapten al estudiante como individuo social a su entorno social, lo que demanda una conducta humana influida por imperativos económicos y culturales (Throsby, 2001). Ante ello, preexiste un engranaje de ideologías económicas y una educación tradicional en la formación profesional del profesorado que promueven un sistema de prácticas arraigadas al funcionamiento del modelo neoliberal (enfoques pedagógicos tradicionales), que aseguren el capital humano pertinente a las necesidades socioeconómicas; esto se puede analizar en el capital cultural del profesorado.

La inclusión educativa requiere de prácticas sociales y culturales que pudieran contravenir prácticas educativas encarnadas en enfoques pedagógicos tradicionales (conservadores) instituidos por el sistema neoliberal en el que se establece un modelo pedagógico que valora la formación de una mano de obra calificada (Puelles, 2009). En consecución a las prácticas sociales y culturales, las prácticas pedagógicas arraigadas en el currículo delimitan la cultura institucional y la cultura escolar (Sacristán, 1996), estas prácticas confluyen en procesos homogeneizantes de la educación (Delgado, 2004), luego entonces, se reflexiona acerca de la importancia del capital cultural del profesorado en los procesos de inclusión educativa.

¿Cuál es la importancia del capital cultural del profesorado en la educación? Bourdieu y Passeron (1996) señalan que el capital cultural es la acumulación de cultura propia de una clase social, la cual es heredada o se adquiere durante el proceso de socialización. Las expresiones del capital cultural se dan en las prácticas culturales que tienen las personas, en el caso de la docencia, las prácticas culturales son comprendidas como producto de interiorizaciones de los contextos de formación educativa que son provistas inconscientemente en esquemas de cognición, afecto y valores. Entonces, el capital cultural del profesorado es todo el bagaje de información y comprensión (que incluye comportamientos e ideologías) que redunda en sus prácticas educativas.

El capital cultural del profesor es de importancia al establecerse las relaciones sociales en el contexto de formación educativa, la incidencia del profesor en el estudiante va a ser regulada por el comportamiento y creencias que este tiene sobre la formación educativa, de tal manera que el acto de enseñanza es una acción pedagógica que se implementa en los aprendizajes. Bourdieu y Passeron (1996) refieren que "toda acción pedagógica es objetivamente una violencia simbólica en tanto que imposición, por un poder arbitrario, de una arbitrariedad cultural" (p. 45).

Con base en lo anterior, la acción pedagógica implícitamente es una violencia simbólica en la que las condiciones culturales son impuestas al estudiante y estas pueden conformar ideologías y comportamientos no deseados en los procesos educativos, 
propiamente inclusivos. En continuación a la teoría de Bourdieu y Passeron (1996), la autoridad pedagógica es una imposición arbitraria que se construye por la acción pedagógica, en este proceso se legitima el acto pedagógico; en relación a la figura del profesor, esta se asume como una figura ortodoxa para la formación profesional del profesorado en la cual se articulan de manera tácita los conocimientos, valores y actitudes. Al respecto, Bourdieu y Passeron (1996) señalan:

En tanto que poder arbitrario de imposición que, por el solo hecho de ser ignorado como tal, se halla objetivamente reconocido como autoridad legítima, la autoridad pedagógica, poder de violencia simbólica que se manifiesta bajo la forma de un derecho de imposición legítima, refuerza el poder arbitrario que la fundamenta y que ella disimula [p. 53].

La autoridad pedagógica del profesorado constituye significados sociales y culturales en las conductas del estudiante, comportamientos, pensamientos, formas de ser; el profesor instituye un habitus en los estudiantes, es decir, se crean disposiciones de estos hacia las prácticas culturales de la escuela. Martínez (2017) elucida el concepto habitus de Bourdieu de la siguiente manera:

El concepto de habitus da un paso más allá del simple hábito (Bourdieu 1984: 268). Es un conjunto de principios de percepción, valoración y de actuación debidos a la inculcación generada por el origen y la trayectoria sociales. Estos principios generan tanto disposiciones como hábitos característicos de dichas posiciones, sincrónicas y diacrónicas, en el espacio social, que hacen que personas cercanas en tal espacio perciban, sientan y actúen de forma parecida ante las mismas situaciones y cada uno de ellos de forma coherente en distintas situaciones. En cierta manera, podemos entenderlo como un genotipo propio de la posición en el espacio social, siendo el fenotipo los hábitos y las prácticas observadas. Además, el habitus es sistemático, en sentido estructuralista; es decir, solo se puede entender su sentido si se relacionan entre sí los distintos hábitos y predisposiciones para la acción de una misma persona, o los habitus entre distintas personas [p. 2].

La escuela como campo (espacio social) constituye una estructura estructurada ya que define el comportamiento de profesores y estudiantes, de cierta manera se conforma la percepción social de actos y singularmente de los conocimientos y aprendizajes. El habitus establece una red de relaciones perceptivas en profesores y estudiantes que incide en la formación del capital cultural y en las determinaciones simbólicas del campo, así, el capital cultural se encuentra ante el estado de prácticas culturales, propiamente en la construcción significativa de experiencias vividas en la escuela, es decir, el habitus como estructura estructurante, por tanto, el capital cultural determinará las prácticas distintivas del profesorado y de estudiantes. Sin embargo, el capital cultural del profesorado representaría una limitante para los procesos de inclusión, esto porque la práctica educativa de profesores está encarnada en experiencias profesionales adquiridas durante su trayecto formativo que influyen e inciden en su acción pedagógica; tanto el conocimiento, costumbres, rutinas, se instituyen en una pedagogía conservadora, en un esquema pedagógico tradicional que se limita a una 
educación bancaria (Freire, 2017) y no a implementar la consciencia inacabada de lo humano (Freire, 2019).

Desde el sentido de la pedagogía conservadora, el profesorado desarrolla una educación depositaria de conocimientos, sin sentido para el estudiante (Freire, 2017), sustenta dicha práctica desde su capital cultural que asegura la estructura del conocimiento universal y la tecnificación de los procesos educativos; en consecuencia, el profesorado desarrolla una pedagogía que instituye prácticas reproductivas en las cuales se entrega el conocimiento como objeto de una realidad dada e idealizada desde el currículo (Bourdieu y Passeron, 1996). El profesorado en su enseñanza omite prácticas sociales democráticas porque la arbitrariedad cultural implica la posición social del profesorado al estructurar el conocimiento como fuente de su acción pedagógica, de tal forma que ostenta un discurso sin trascendencia sobre la alteridad y diversidad de los estudiantes, únicamente importa el componente psicológico y cognitivo, de esta forma se construye la ritualidad del discurso pedagógico como canon de la práctica educativa, "el discurso educativo conservador presenta a menudo una visión de la cultura y el conocimiento en que ambos se abordan como partes de un depósito de artefactos constituidos como canon" (Giroux, 2003, p. 177).

Las prácticas educativas conservadoras se encuentran mediadas por la homogeneización de la enseñanza, la generalidad de estrategias didácticas y actividades pedagógicas rutinarias como el diario pase de lista, solicitar silencio constantemente, evaluaciones periódicas con los mismos instrumentos, aplicar las mismas dinámicas de aprendizaje, esto es, sincretizar al estudiante en la visión disciplinar del currículo, aun cuando en el currículo pudiera señalar la multiculturalidad, las diferencias o valores afines a la inclusión, en el aula las prácticas culturales promueven un discurso antagónico a las prácticas inclusivas porque el capital cultural del profesorado se conformó desde un sistema hegemónico enajenante a los procesos inclusivos y su actuación pedagógica dista de las necesidades educativas.

Giroux (2003) refiere que el discurso provoca y legitima las configuraciones de tiempo, espacio y narrativa, se da privilegio a ciertas versiones de la ideología, al comportamiento y a la representación de la vida cotidiana. "Como «tecnología del poder», el discurso adquiere una expresión concreta en las formas de conocimiento que constituyen el currículum [sic] formal, así como en la estructuración de las relaciones sociales del aula que constituye el currículum oculto de la enseñanza" (p. 176). Si el capital cultural del profesor se encuentra encarnado en una pedagogía y discurso conservador conforme a la lógica del sistema hegemónico, las relaciones sociales del aula estarán también dadas en esa arbitrariedad cultural. Contrario a las prácticas educativas encarnadas, Skliar (2017) propone la necesidad de un lenguaje nuevo en la educación que pueda evidenciar la normalización, el utilitarismo y consumo que 
atañe a la educación, de igual manera explorar las diferencias, propiamente, la distinción entre diferencia y diversidad, esta propuesta es la pedagogía de las diferencias.

Skliar (2017) plantea la acción educativa que provoque el encuentro en el aquí y ahora, de considerar a estudiantes que abandonan el currículo (como forma total de encuentro con el mundo) y sustituir la meritocracia escolar; Skliar argumenta que las instituciones educativas desvalorizan el presente al considerar el progreso y la formación laboral, el presente como tiempo de existencia del cual se significa el mundo y la vida; para él, la enseñanza es una provocación al pensamiento en el que se constituye el acto pedagógico como responsabilidad, y en esa conformación de sentido de responsabilidad se valora, visibiliza al otro que aprende; en este proceso hay distintos signos y sentidos, educar es un acto de inclusión, de legitimación de la existencia.

La pedagogía de las diferencias está estructurada en la alteridad, la cual tiene en la conversación compleja de la otredad su valía. La conversación con el otro es reconocer la existencia del otro, esto es, que hay un pensar sobre el otro, el cual es responsabilidad del acto pedagógico; esto conlleva analizar la normalidad del mundo estructurado en el currículo, en lo educativo; dicha normalidad se instaura o se regula desde el hábito, por lo que las prácticas culturales del profesorado tendrán que imposibilitar el mundo del estudiante como una situación natural, esto, es adjudicar la normalidad concerniente a la visión de mundo neoliberal. Es posible enunciar que el capital cultural del profesorado se encuentra malversado en la normalidad del mundo, y se ha consentido una idea de lo diverso alienado a los otros. El pensar en el otro no es simplemente una forma reproductiva o mecánica sino que sobrelleva a la capacidad de participar en el encuentro con el otro, en una conversación cercana con los otros.

Ante la resistencia del capital cultural del profesorado por otrora formación profesional, se tiene que generar una ruptura epistemológica y conceptual de pedagogías conservadoras y tradicionales, esto exige una consciencia del inacabamiento de lo humano (Freire, 2019). Se tiene que asumir una enseñanza crítica que posibilite la propia producción o construcción, porque enseñar no se reduce a la transferencia de contenidos, sino que requiere de la extensión; en el caso de la inclusión como significado abstracto el estudiante estará envuelto de la inclusión, esto conlleva pensar que la enseñanza es en esencia pensar acertadamente, lo que es difícil porque se tiene que evitar el simplismo, las facilidades, las incoherencias burdas (Freire, 2019).

Las prácticas inclusivas se suscitarán cuando el profesorado cambie el paradigma de la pedagogía conservadora hacia una pedagogía de las diferencias; la dificultad de implementar la inclusión educativa en las prácticas culturales estará en medida de la comprensión del espacio social de la escuela. La escuela es el medio social o campo que se tendrá que reflexionar como factor de propiciación de procesos inclusivos. 


\section{LA ESCUELA, ¿ESPACIO SOCIAL DE INCLUSIÓN O EXCLUSIÓN?}

La escuela es un espacio social en el que convergen y conviven administrativos, profesorado y estudiantes; en la escuela se crea la cultura escolar a través de la socialización. La escuela es el espacio social en el que el capital cultural del profesorado cobra sentido en razón de la función social y cultural que tienen los profesores, es el lugar en el que se dan las estructuras estructuradas de las prácticas educativas. Es un espacio de interacción social en el que se construye la identidad; una escuela inclusiva implica la comprensión de cómo los profesores conciben la escuela, porque es ingenuo pensar que la escuela es únicamente el espacio social y cultural en el que se construyen conocimientos.

La inclusión educativa en la escuela implica la responsabilidad de directivos y profesorado de intervenir en mecanismos de exclusión y segregación que se susciten, para ello, es pertinente la continua reflexión de la práctica educativa, establecer criterios de prevención y solución a los problemas sociales; de igual manera se tiene que considerar la participación de los padres de familia y orientar el fin de la escuela hacia al enfoque de la diversidad y sus diferentes acepciones: social, cultural, étnica, religiosa, de género, lingüística, etc.; es de suma importancia diseñar estrategias que involucren la participación social de todos y fomentar prácticas sociales democráticas.

El modelo de la escuela inclusiva propone una educación integral y de calidad para todos sin importar las características personales y las necesidades de apoyo de los estudiantes (Blasco, 2018). Abordar la educación inclusiva es pensar en "todos", lo cual es visibilizar lo que el otro es, no lo que se atribuye socialmente al otro, porque se piensa en el discapacitado, no en su persona; en la escuela se forman prejuicios religiosos, pero no se valora la cosmovisión espiritual; por consiguiente, el ideal de la escuela inclusiva es la total apertura a la diversidad. La escuela inclusiva visibiliza las necesidades, intereses, pensamientos, emociones y gustos, es decir que las prácticas educativas abordan el principio de otredad. Se piensa entonces en la otredad y alteridad; en la necesidad de reconocer al otro se crea una representación de las diferencias entre culturas, de lo que caracteriza a la persona, su identidad y diferencia. La escuela inclusiva pondera la participación de todos, valora la intersubjetividad y expresiones de identidades.

La escuela que trata la diversidad erige una enseñanza de las diferencias, lo cual conlleva demarcar prácticas culturales excluyentes como la homogeneización de la cultura e identidad; si la escuela genera una opresión hacia el estudiante en la omisión de su identidad cultural, la educación enajenará la realidad social y cultural del estudiante en un escenario al que no pertenece; se mantienen estructuras estructuradas que vuelven compleja la relación de emancipación, no se educa para la libertad (Freire, 2017). La hegemonía es un recurso lógico-instrumental que adecua el sentido de la relación entre identidad y escuela (Bravo, Peña y Jiménez, 2006). 
¿Qué significados y comportamientos habrían de establecerse para apuntalar la inclusión en la escuela? En relación a la cultura escolar, ¿cómo desarraigar la intolerancia, la discriminación, la indiferencia y la violencia de la escuela que se erige en la práctica educativa? Toda resistencia apegada a pedagogías conservadoras pugnará por la individualidad y el establecimiento de estrategias didácticas generalizadas y de enseñanzas antagónicas a la construcción de las otredades y alteridades.

López (2001) enuncia que la escuela constituye un punto sociocultural en donde se construyen identidades, es el lugar en el cual cohabitan las subculturas y culturas; tradiciones, costumbres, rutinas, rituales matizan las identidades y entretejen las interrelaciones de las prácticas sociales y culturales de las actividades pedagógicas; la cultura institucional en la perspectiva de la teoría crítica se ordena y se instaura desde la cultura dominante, esta cultura se concreta en el currículo como un sistema ideológico y pedagógico de forma racional en la práctica pedagógica.

Apple (2008) plantea que el aspecto racional del currículo se encuentra organizado por los aspectos económico, político, ideológico e intelectual; el capital cultural del profesorado induce ideologías y comportamientos que ha adquirido en su trayecto formativo; las prácticas educativas están encarnadas en la racionalidad del currículo porque el profesor apega su práctica educativa a las propuestas didácticas de enseñanza; el currículo instituye un deber ser en la escuela, produciendo un paralelismo entre lo institucional y lo académico. El inconveniente de inclusión en la escuela es que no se tenga consciencia del habitus construido en las prácticas sociales y culturales de las instituciones educativas, pues las prácticas educativas confluyen a través de la educación (Sacristán, 2005). Entonces, la escuela es un mecanismo sociocultural de exclusión si no se cambia la concepción de escuela, en otras palabras, modificar el capital cultural de las prácticas educativas que se tienen en las escuelas.

La escuela pensada como un espacio social de exclusión promueve la subjetivación del estudiante en cuanto a determinantes administrativos y normativos: estudiantes que no cumplan con las características o requisitos del currículo serán excluidos (normativo) y quienes no cuenten o adquieran las características culturales y de aprendizaje que provee la escuela tendrán un rechazo desde lo institucional (administrativo); sin embargo, el estudiante resiste al capital cultural de la escuela. Bourdieu (1979, citado en Brunet y Morell, 1998) refiere que la escuela contribuye a reproducir el capital cultural como estructura del espacio social, en otras palabras, la concepción de la escuela surge desde el plano ideológico hegemónico y por consiguiente habría que reflexionar en cómo la acción pedagógica violenta simbólicamente los procesos inclusivos al no considerar las resistencias y contestaciones de estudiantes en el sistema cultural institucionalizado. 


\section{LA EDUCACIÓN OBLIGATORIA, ¿UN INSTRUMENTO NEOLIBERAL DE EXCLUSIÓN SOCIOEDUCATIVA?}

El hombre que quiere ser sujeto de la historia debe crear sentido desde reconocer los espacios para ser sujeto, pensado en, desde y para la historia (Zemelman, 2002); el profesorado, para crear sentido de su realidad educativa y no subjetivarse a una pedagogía conservadora, precisa de reflexionar los procesos de su práctica educativa; su práctica remite su formación académica que evidencia una postura de pensamiento, y un primer atisbo de la práctica educativa es el componente político-pedagógico de la educación obligatoria.

La educación obligatoria deviene de políticas educativas y pedagogías pertinentes al modelo económico, su fin es la escolarización de las sociedades y provee una racionalidad instrumental de la enseñanza. Giroux (2004) señala que se han considerado las teorías de la reproducción para abordar la formación social necesaria que mantiene las relaciones capitalistas de producción y en la que las escuelas ocupan un papel esencial. Las teorías de la reproducción se orientan en cómo el poder y el control se utilizan para beneficio de la sociedad dominante dentro y fuera de la escuela, ante lo cual,

La escolarización representa el sitio social principal para la construcción de subjetividades y disposiciones, y un lugar donde los estudiantes de diferentes clases sociales aprenden las habilidades necesarias para ocupar sus lugares específicos de clase en la división ocupacional del trabajo [Giroux, 2004, p. 109].

La escolarización es una condición social y pedagógica de las sociedades modernas en que las etapas de desarrollo del estudiante se han categorizado. La escuela escolariza la niñez, adolescencia y juventud a través de los niveles educativos de los sistemas sistema de educación oficial; la escuela es el lugar que fragua el habitus, impone la ideología y hegemonía del sistema neoliberal. La educación obligatoria como hecho educativo de la escolarización enjuicia la satisfacción de formación académica y las necesidades de infantes, adolescentes y jóvenes en la escuela y sociedad, promoviendo un estatus moral de ser escolarizados.

Si bien Giroux (2004) retoma las concepciones de Althusser en cuanto a la superestructura de la teoría marxista, se argumenta que la base económica de las instituciones de la sociedad no se simplifica a determinaciones de causa-efecto, por el contrario, la legitimación de las sociedades capitalistas industrializadas ocurre en la autorregulación del Estado que domina mediante la fuerza y se representa por aparatos represivos (ejército, policía, corte, prisiones y aparatos ideológicos), por ende, la escolarización es una forma de represión de las sociedades. La escolarización contribuye a la formación de capital cultural del profesorado porque es un sistema ideológico y se confina en relaciones estructuradas estructurantes dado que la educación es continua y progresiva y de carácter legal obligatorio. 
La pedagogía como ciencia de la educación mantiene una ambivalencia entre la esperanza en las condiciones futuras del estudiante y de zozobra sobre poblaciones asumidas como peligrosas por estigmatizaciones del propio sistema neoliberal, porque así se determinan en la posición político-pedagógica de escolaridad; dicho de otra manera, hay una demarcación de carácter económico (mercantilista) en sociedades escolarizadas y no escolarizadas; es pertinente una pedagogía crítica que emancipe los procesos de opresión en la educación (Freire, 2017). La educación, para implementarse formalmente, se legitima en la escuela por un marco legal y cultural que valida socialmente su institucionalidad. La escuela se encuentra en un sistema que pertenece al Estado, el cual estableció la obligatoriedad de la educación; hay elementos como la alfabetización, expansión de derechos educativos, educación de calidad, entre otros, que fundamentaron políticas públicas tendientes a mejorar las condiciones sociales, económicas y culturales de una sociedad bajo el predominio de un sistema ideológico que caracterizó a la escuela como un aparato de inculcación ideológica de las clases dominantes (Pineau, Dussel y Caruso, 2015).

Hebrard (1989, citado en Pineau, Dussel y Caruso, 2015) enunció que la educación obligatoria consolidó la institucionalización de la escuela suscitando los saberes básicos de las sociedades, con ello la implementación de la escolaridad, por lo que la escolarización adquirió un estatus moral en los sujetos para incluirse en el sistema social productivo. La escolarización de las masas es el ideal de las sociedades modernas, es una condición del progreso material y espiritual (Sacristán, 2005). Escolarizar suele relacionarse con educar, instruirse, aunque educar no es precisamente escolarizar, educar es una tarea social, de consciencia sobre las necesidades de lo humano, necesidades que en el esquema filosófico neoliberal son determinadas al margen de lo económico, ante lo cual se cuestiona si el capital cultural crea en los estudiantes consciencia de ser sujetos de inclusión.

Lundgren (1983, citado en Kemmis, 2008) indica que las sociedades se organizan para asegurar el conocimiento sobre las generaciones futuras; la escolarización se conforma como una cultura de la educación, esto culturaliza al estudiante como sujeto escolarizado, un sujeto pertinente y necesario para sociedades mercantilizadas; la escolarización (implica el conocimiento de generación a generación) como situación social institucionalizada asegura los fines educativos a través de prácticas pedagógicas tácitas y arbitrarias instituidas desde el currículo (propiamente el desarrollo de pedagogías conservadoras) e implementa las políticas neoliberales al escolarizar al sujeto.

La escolarización es un hito cultural tendencioso de las clases sociales en la mecánica social de educarse para mejorar la calidad de vida. Rivero (2013) enuncia que la escuela preconiza prácticas pedagógicas delimitadas por sistemas que conforman una totalidad socio-político-económica y cultural a través de procesos como la globalización. Paradójicamente, la ideología neoliberal se nutre de la escolarización e implícitamente construye el habitus del estudiantado. 
La escolaridad de las sociedades está determinada por el factor económico que encauza el detrimento de la deshumanización al priorizar y valorar las habilidades y conocimientos concernientes a la productividad; históricamente la inteligencia se delimitó al campo de las competencias. La escolarización obligatoria fue un modelo iniciado en el siglo XIX y principios del siglo XX con el fin de satisfacer las necesidades sociales y económicas (Molina, 2007); es un factor que determina el avance social desde factores interdisciplinarios del currículo; la tendencia del enfoque económico es medir la rentabilidad del sistema de enseñanza para la colectividad (Pansza, 2003). Desde el currículo las personas son insertadas en la sociedad bajo la lógica de la educación obligatoria, en la cual la representación de escolarización es una construcción mental, una representación colectiva (Sacristán, 2005).

A partir de la obligatoriedad de la educación en las sociedades se fecundó una imagen de mejora social, este aspecto de mejora se ofertó mediante el estado de bienestar (Sacristán, 2005), por lo que la relación entre currículo y sociedad sopesa el hecho de la formación de estudiantes capacitados desde una educación socioeconómica y tecnológica (capital humano). Por tanto se caracteriza a la educación como un bien o necesidad social, por lo que la educación resulta ser pragmática y funcional, características opuestas al humanismo de la inclusión educativa.

Como se ha analizado, la escolarización resulta una justificación política de la economía, por lo que educativamente se excluye a quien no está escolarizado, en otras palabras, quien no desarrolle determinadas competencias; se enuncia al mismo tiempo una clasificación de lo ideal, esto es, un estudiante cosificado por sus habilidades y conocimientos, y no se valora el ser de lo humano. En contrasentido a lo escolarizado, el estudiante atribuido socialmente como desescolarizado implica un esquema de funcionalidad dentro del sistema económico y mercantil, por lo cual el desescolarizado, en ciertos casos tipificado de analfabeta, es consecuente de una disfunción del sistema educativo y el sistema económico laboral (un error del sistema), luego entonces, no es apropiado ni incluido socialmente por su insuficiencia de capital cultural y, desde lo pedagógico-económico, su capital humano.

\section{ConClusión}

El capital cultural del profesorado se encarna en pedagogías conservadoras y se instituye en prácticas educativas que complican la inclusión educativa; la escuela y escolaridad obligatoria son elementos pedagógicos que contribuyen a inhibir los procesos de inclusión educativa. Los sistemas educativos bajo el enfoque neoliberal promueven prácticas sociales y culturales de exclusión y segregación, esto continuará si no se reflexiona el capital cultural del profesor y el habitus construido en los estudiantes. Ante la irreflexión de la pedagogía conservadora que aborda los discursos de 
inclusión es eminente una pedagogía de las diferencias que reflexione las vivencias del aula y la diversidad, una pedagogía que considere la otredad y la alteridad.

Las prácticas educativas bajo el esquema de una pedagogía conservadora hallarán una demarcada línea condicionada en la hegemonía ideológica del sistema neoliberal, la pedagogía tradicional no cuestiona, es silenciosa y reproductiva de la cultura dominante, por tanto es conveniente a intereses particulares. Es necesario generar rupturas ideológicas y conceptuales para repensar el acto pedagógico que establece el profesorado en su práctica educativa.

En la perspectiva de la escuela como espacio sociocultural de exclusión se requiere replantear las prácticas culturales que limitan las expresiones de la diversidad; si bien la escuela construye identidades, es oportuno visibilizar los mecanismos socioculturales de identidad puesto que se construye un tipo de sujeto para los propósitos del sistema educativo neoliberal. Al considerar la pedagogía crítica se atribuirá a la acción pedagógica una pedagogía de las diferencias que visibiliza al otro y promueve la otredad, alteridad e inclusión, elementos contrarios a las prácticas educativas confinadas a la exclusión y segregación; la pedagogía crítica denotará el influjo de la educación como conformación de un modelo económico mercantil que selecciona y clasifica las competencias (habilidades y conocimientos).

Por último, la concepción de la educación obligatoria como política social de escolarización es una condición política-pedagógica del sistema económico que instrumenta la instrucción, capacitación y formación del capital humano. La obligatoriedad y universalización de la educación constituyen la escolarización de las sociedades modernas, estudiantes que no cumplan con las políticas de escolarización serán excluidos, segregados, insuficientes al sistema social económico; la desescolarización tiene un sentido funcional (capital humano) en el sistema neoliberal, por tanto la educación resulta un mecanismo neoliberal de exclusión socioeducativo si no se cambian las prácticas educativas apegadas y encarnadas en pedagogías conservadoras, tradicionales, que deshumanizan al sujeto (Freire, 2017). Queda el hilo abierto a posteriores reflexiones sobre el capital cultural y su correlación con la escuela y educación obligatoria, ya que la inclusión educativa es un modelo pedagógico que se encuentra en las agendas políticas de organizaciones públicas y organismos no gubernamentales y demanda su aplicación en los diferentes sistemas educativos internacionales ante la crisis y vulnerabilidad de la educación.

\section{REFERENCIAS}

Apple, M. (2008). Ideología y currículo. Madrid: Akal, S.A. Bartolomé, M., y Cabrera, F. (2007). Construcción de una ciudadania intercultural y responsable. Madrid: Narcea.

Beyer, L., y Liston, D. (2001). El currículo en conflicto. México: Akal.
Blasco, P. (2018). Respuestas a la diversidad en la escuela inclusiva. De la teoría a la práctica. Valencia: Nau Libres. Bourdieu, P., y Passeron, J.C. (1996). La reproducción, elementos para una teoría dela enseñanz̧a. Barcelona: Fontamara S.A. 
Bravo, H., Peña, S. L., y Jiménez, D. A. (2006). Identidades, modernidad y escuela. Bogotá: Universidad Pedagógica Nacional.

Brunet, I., y Morell, A. (1998). Clases, educación y trabajo. Madrid: Trotta.

De la Torre, M. (2004). Del humanismo a la competitividad. México: Universidad Nacional Autónoma de México.

Díaz, F. (2002). Didáctica y currículo: un enfoque constructivista. Cuenca: Ediciones de la Universidad de Castilla.

Dueñas, M. (2010). Educación inclusiva. Revista Española de Orientación y Psicopedagogía, 21(2), 358366. Recuperado de: https://www.redalyc.org/ pdf/3382/338230785016.pdf.

Freire, P. (2017). Pedagogía del oprimido. México: Siglo XXI.

Freire, P. (2019). Pedagogía de la autonomía. Saberes necesarios para la práctica educativa. México: Siglo XXI.

García, R. (2006). Innovación, cultura y poder en las instituciones educativas. España: Centro de Investigación y Documentación Educativa.

Giroux, H. (2003). Pedagogía y politica de la esperanza: teoría, cultura y enseñanza: una antología crítica. Buenos Aires: Amorrortu.

Giroux, H. (2004). Teoría y resistencia en educación. México: Siglo XXI.

Giroux, H. (2006). La escuela y la lucha por la cindadania, Pedagogía crítica moderna. México: Siglo XXI.

Kemmis, S. (2008). El currículum: más allá de la teoría de la reproducción. Madrid: Morata.

López, M. (2001). La escuela por dentro. Perspectiva de la cultura escolar en la España del siglo XXI. Valencia, España: Universitat de Valencia.

Martínez, B. (1998). Proyectos curriculares y práctica docente. Sevilla, España: Díada Editora.

Martínez, R. (2014). Educación, neoliberalismo y justicia social. Una revisión crítica del desarrollo humano desde la carta de la tierra y la economía social. Madrid: Pirámide.

Martínez, J. (2017). El habitus. Una revisión analítica. Revista Internacional de Sociología, 75(3) 1-14. Recuperado de: http://revintsociologia.revistas.csic.es/index.php/ revintsociologia/article/view/680.
Molina, S. (2007). La escolarización obligatoria en el siglo XXI. Madrid: La Muralla S.A.

Montanchez, M. (2015). La educación como derecho en los tratados internacionales: una lectura desde la educación inclusiva. Revista de Pary Conflictos, 8(2) 243-265. Recuperado de: https://revistaseug.ugr. es/index.php/revpaz/article/view/3297.

Pansza, M. (2003). Pedagogía y currículo. México: Gernika.

Pineau, P., Dussel, I., y Caruso, M. (2015). La escuela como máquina de educar, tres escritos sobre un proyecto de modernidad. Buenos Aires: Paidós.

Puelles, M. (2009). Globalización, neoliberalismo y educación. Avances en supervisión educativa. Revista de la Asociación de Inspectores de Educación de España, (11), 1-12. Recuperado de: https://avances.adide.org/ index.php/ase/article/view/429/273.

Rivero, R. (2013). Educación y pedagogía en el marco del neoliberalismo y globalización. Perfiles Educativos, 35(142), 49-166. Recuperado de: http://www. scielo.org.mx/pdf/peredu/v35n142/v35n142a10. pdf.

Sacristán, G. J. (1996). El curriculum: una reflexión sobre la práctica. Madrid: Morata, S.L.

Sacristán, G. J. (2005). La educación obligatoria y su sentido educativo y social. Madrid: Morata.

Sánchez, C. (2018). La inclusión educativa como proceso en contextos socioeducativos. Madrid: Universidad Nacional de Educación a Distancia.

Skliar, C. (2017) Las pedagogías de las diferencias. Buenos Aires: Noveduc.

Torres, J. (2007). La educación en tiempos de neoliberalismo. Madrid: Morata.

Throsby, D. (2001). Economía y cultura. Cambridge.

Villalobos, G., y Pedroza, R. (2009). Perspectiva de la teoría del capital humano acerca de la relación entre educación y desarrollo económico. Tiempo de Educar, 10(20), 273-306. Recuperado de: https:/ / www.redalyc.org/articulo.oa?id=31112987002.

Zemelman, H. (2002). Necesidad de consciencia. Un modo de construir conocimiento. México: Anthropos.

Cómo citar este artículo:

Ortiz Huerta, M. G., y Zacarías Gutiérrez, M. (2021). El capital cultural del profesorado en los procesos de inclusión educativa. IE Revista de Investigación Educativa de la REDIECH, 12, e1166. doi: 10.33010/ie_rie_rediech.v12i0.1166. 